\title{
Limeños y arequipeños ante la diáspora venezolana: representaciones estéticas y de comportamiento
}

\author{
Luis Andrade Ciudad, Anahís Samamé Rispa, \\ Sergio Lazarte Chicata, Claudia Crespo del Río \\ y Pamela Jimenez Lizama
}

Desde el análisis del discurso, nos aproximamos a las representaciones sobre la masiva migración venezolana en el Perú. Examinamos un corpus de 64 entrevistas semiestructuradas realizadas a residentes de Lima y Arequipa. Observamos los patrones en los discursos de los entrevistados teniendo en cuenta su localidad y su tipo de ocupación. Nos concentramos en las representaciones sobre la estética de los migrantes y sobre su comportamiento. Respecto a las primeras, resalta la caracterización de estos como altos, en el caso de los hombres, y voluptuosas, en el caso de las mujeres, así como la existencia de una cultura de la belle$z a$ valorada ambivalentemente. En cuanto a las segundas, se observaron caracterizaciones negativas, como ser bulleros o soberbios, pero también positivas, como ser amables y alegres. Nuestros hallazgos sugieren la conveniencia de integrar a las aproximaciones sociolingüísticas sobre la migración los planteamientos sobre las estrechas relaciones entre el lenguaje y los fenómenos de racialización, así como las pautas más clásicas sobre la construcción de la identidad en interacción, derivadas de la lingüística sociocultural.

Palabras clave: diáspora venezolana, raza, discurso, Lima, Arequipa

Limeños and arequipeños facing the Venezuelan diaspora: Aesthetic and behavioral representations. With discourse analytical tools, this paper addresses emergent discourses related to the massive Venezuelan migration to Peru. We examine a corpus of 64 semistructured interviews carried out in Lima and Arequipa with Peruvian dwellers of both cities. Common discourse patterns and differences are identified, both among interviewees of both cities, and among interviewees that perform basic and non-basic occupations. We focus on two aspects. The first one relates to representa- 
tions of the migrants' physical features and personal care, where qualifications such as tall or voluptuous, and references to a beauty culture stand out. The second aspect includes representations of their social behaviour, with adjectives like noisy, arrogant and friendly and cheerful. Our findings suggest the need of enriching sociolinguistic approaches to migration with recent claims about the tight relationship between language and racialization phenomena, as well as with more classic guidelines for analysing identity in interaction, derived from sociocultural linguistics.

Keywords: Venezuelan diaspora, language, race, Lima, Arequipa

\section{Introducción: migración, lenguaje y diferenciación social}

La sociolingüística de la migración o de la movilidad considera, actualmente, los fenómenos de globalización y la alta movilidad de las personas en las sociedades contemporáneas (Blommaert 2010). En los últimos años, los estudios socioeconómicos sobre los procesos migratorios han tomado en cuenta las características particulares de las migraciones producidas entre países del Sur global (migraciones Sur-Sur), tanto desde el punto de vista de las motivaciones de los migrantes para dejar el lugar de origen (León y Falcón 2016) como desde las dificultades específicas de integración que estos deben afrontar en los países de destino (OECD 2011).

Proponemos que, para observar un proceso de migración masiva Sur-Sur desde un punto de vista discursivo, la sociolingüística de la migración puede enriquecerse con los recientes planteamientos sobre las estrechas relaciones entre el lenguaje y los procesos de racialización (Rosa y Flores 2017; Zavala y Back 2017), así como con las pautas más clásicas sobre la construcción de la identidad en interacción derivadas de la lingüística sociocultural (Bucholtz y Hall 2005). Esta integración puede ser particularmente útil para contextos poscoloniales como las sociedades latinoamericanas (Quijano 1992, 2014).

Aprovechamos, así, conceptos desarrollados en este marco, como el de 'habla racial', que permite conectar el comportamiento lingüístico a la raza, enmarcando los comentarios evaluativos de los hablantes acerca de los vínculos entre ambas dimensiones (Anderson 2008: 108), muchas veces sin hacer alusión directa a los aspectos raciales (Zavala y Back 2016: 22). Asimismo, aludimos al 'enregistramiento', inicialmente definido como el conjunto de "procesos a través de los cuales un repertorio lingüístico se vuelve diferenciable dentro de una lengua como un registro de formas 
socialmente reconocido” (Agha 2003: 231, traducción nuestra) y que, en una reformulación reciente, se ha vinculado a la naturalización del nexo entre formas lingüísticas y modelos de persona (Rosa y Flores 2017: 631).

De este modo, categorías estándares para observar las estrategias discursivas en torno a la representación de los actores y los procesos-como modalidad (Palmer 2001; Wiemer 2018), foco y estructura informativa (Ferrari, Giammatteo y Albano 2011), mitigación y atenuación (Albelda Marco 2018; Briz 2006)- pueden generar aproximaciones productivas engarzándose en una comprensión situada de la construcción de la diferencia (Irvine y Gal 2000) y, en última instancia, de las nuevas jerarquías establecidas entre los grupos migrantes y las sociedades receptoras (Kesselheim 2007).

Aplicamos este enfoque para identificar y analizar los discursos emergentes sobre la masiva migración venezolana al Perú en los últimos años. Si bien ha habido aproximaciones sociológicas y sociojurídicas a esta diáspora, no existen hasta el momento estudios sobre los discursos que están surgiendo entre peruanos y peruanas al respecto. Después de contextualizar brevemente la migración venezolana al Perú (sección 2), explicamos la metodología (sección 3), para luego presentar los resultados referidos a representaciones del físico y el cuidado personal (sección 4), y los que atañen a los rasgos de comportamiento (sección 5). En la sección 6, discutimos estos hallazgos en función del enfoque teóricoconceptual presentado y, en la sección 7, resumimos las conclusiones.

\section{Contexto: la diáspora venezolana en el Perú}

Los problemas socioeconómicos, políticos y de seguridad pública que afronta Venezuela en los últimos años han obligado a más de un millón de venezolanos y venezolanas a dejar su país, en una diáspora que ha sido considerada como la historia con mayúsculas de Sudamérica en lo que va del siglo XXI (Agencia EFE 2018). Hasta febrero de 2020, más de 860000 migrantes venezolanos habían sido recibidos en el Perú, segundo país en albergar la mayor cantidad de población venezolana (Organización Internacional para las Migraciones 2020: 6). Debido a este elevado número, Loayza (2020: 179) considera que se trata del "suceso migratorio más impactante en el devenir de la historia peruana".

Según los datos recogidos por la OIM de septiembre a diciembre de 2019, las y los migrantes venezolanos en el Perú procedían principalmente de Caracas-Distrito Capital y Carabobo, con $17 \%$ y 13,1 \% del total de encuestados, respectivamente (Organización Internacional para 
las Migraciones 2020: 12). Estas dos ciudades se caracterizan por ser norcosteñas y colindar con el mar Caribe. Con menores porcentajes, la procedencia de los migrantes era de ciudades como Aragua, Zulia, Lara, entre otras. La misma organización informa que gran parte de esta población se encontraba en el rango de edad de entre 18 y 29 años $(45,5 \%)$, y la mayoría eran mujeres (55,2\%). Además, en cuanto al nivel educativo, el 55,5\% contaba con estudios secundarios y el 30,7 \%, con estudios universitarios (Organización Internacional para las Migraciones 2020: 8-9).

En el Perú, estos migrantes han accedido mayoritariamente a ocupaciones básicas en el sector servicios y muchos trabajan en la informalidad: en 2019, solo $7 \%$ de los que formaban parte del mercado laboral local eran asalariados (Koechlin et al. 2019: 66). Además, Blouin reporta el alto porcentaje (46\%) de personas que trabajan en el comercio ambulatorio (2019: 42). Tomando en cuenta que la informalidad es una característica saltante del mercado laboral peruano y que la mayor cantidad de migrantes se sitúa en este sector laboral (Blouin 2019: 43), nos pareció relevante observar las diferencias discursivas entre trabajadores de ocupaciones básicas y no básicas.

Al ser el Perú tradicionalmente polo de emigración y no destino de inmigrantes, es previsible que una diáspora de la magnitud señalada esté generando discursos de muy distinto signo en la sociedad receptora, una sociedad atravesada por intensas fracturas sociales, étnicas y económicas, y que fue profundamente transformada en el siglo XX por experiencias migratorias internas (Franco 1986; Matos 1984). Enfocamos nuestro problema en dos ciudades que son las más importantes del país en términos demográficos, pero que han desarrollado identidades urbanas muy diferentes: mientras Lima, la moderna capital, es una metrópolis de más de nueve millones y medio de habitantes que ha visto trastocada a lo largo del siglo XX su tradicional identidad costeña por los efectos culturales y sociales de una migración interna procedente de distintos sectores del país y sobre todo de los Andes (Arellano y Burgos 2010), Arequipa alberga a casi un millón de habitantes y, aunque también constituye un polo migratorio y de prestigio para regiones como Puno y Cusco, sus habitantes mantienen la histórica percepción de una Ciudad Blanca construida sobre valores asociados al honor, el trabajo y la decencia (Chambers 2003).

\section{Metodología y datos}

Entre octubre y noviembre del 2019, realizamos 32 entrevistas semiestructuradas en cada una de estas ciudades, y las analizamos tomando en 
cuenta las diferencias entre ellas, y entre los tipos de ocupación laboral de los entrevistados, de acuerdo con las pautas del Instituto Nacional de Estadística e Informática, que nos permitieron distinguir entre empleos de ocupación básica y no básica (INEI 2016).

Para reclutar a los participantes, nos enfocamos en cada ciudad en dos distritos con pronunciada presencia de migrantes: Los Olivos y Magdalena en Lima; Cercado y José Luis Bustamante y Rivero en Arequipa. ${ }^{1}$ La condición principal fue residir o laborar en el distrito en el último año, al margen de que los entrevistados, todos peruanos, hubieran nacido allí. Además, la mitad de los entrevistados desempeñaba ocupaciones básicas como servicios de peluquería y atención en restaurantes; la otra mitad, de ocupación no básica, trabajaba, por ejemplo, en la docencia y en la prestación de servicios de salud. Desde una perspectiva economicista de la migración, se esperaría que los discursos de rechazo se presenten sobre todo entre los primeros (ver sección 2). Aunque el sexo y la edad no estuvieron entre nuestros criterios principales, cuidamos de que la muestra los balanceara adecuadamente.

\section{Resultados: representaciones sobre el físico y el cuidado personal}

Expondremos en esta sección las representaciones identificadas acerca de las características físicas de los y las migrantes venezolanas, por un lado, y sus prácticas de cuidado personal, por otro.

\subsection{Varones altos, mujeres voluptuosas: características físicas}

La evaluación positiva sobre el atractivo físico de los y las migrantes venezolanas es común a las dos ciudades y se presenta tanto en entrevistados de ocupación básica como no básica. El uso de elementos de modalidad categórica es frecuente en los enunciados referidos a este punto, con adverbios como definitivamente y evidentemente, en ocasiones reiterados, y enfatizadores como muy:

(1) Definitivamente, definitivamente son hermosas, son muy bellas, tanto las mujeres como los varones (Arequipa, ocupación básica, Lo8H(29)BRB).

(2) ...las chicas son guapas, evidentemente (Lima, ocupación no básica, $\mathrm{A09H}(44) \mathrm{MNB})$. 
Observando las descripciones físicas con más detalle, notamos un contraste entre la manera como se enfatizan las características de los hombres y de las mujeres venezolanas. Mientras los varones son caracterizados como altos -a veces delgados, a veces fornidos-, las mujeres son descritas como bien proporcionadas y voluptuosas.

(3) Los varones son altos y delgados y las mujeres obviamente también son proporcionadas, son finitas de arriba y proporcionadas de abajo, entonces, es característico de su genética (Arequipa, ocupación no básica, C07M(31)BRNB).

(4) ...la mayoría son más altos, son altos y son un poco más fornidos en su contextura, en su físico; las mujeres también son voluptuosas, también son altas; una buena talla tienen ellos (Arequipa, ocupación básica, L09H(36)BRB).

Algunos entrevistados relacionan estas características con la ascendencia africana que atribuyen a los migrantes. La dimensión racial está presente en el material, pero se encuentra más clara en Arequipa y en el grupo de ocupación no básica. De 32 entrevistados en cada ciudad, 15 arequipeños traen espontáneamente este tema cuando se les pregunta por las características físicas de los migrantes, mientras que solo 4 lo hacen en Lima. De esos 15 arequipeños, 9 son de ocupación no básica y 6 de ocupación básica.

Este contraste puede relacionarse, por un lado, con la menor proporción de población autoidentificada como afroperuana en la provincia de Arequipa (1,92 \%, 16869 personas) que en Lima (2,76 \%, 194661 personas) según el censo del 2017 (Instituto Nacional de Estadística e Informática 2017). ${ }^{2}$ La menor presencia de afroperuanos haría más saltante este rasgo en la percepción que los arequipeños tienen de los venezolanos. Por otro lado, el contraste puede vincularse con la autopercepción histórica de Arequipa como Ciudad Blanca, conformada por sujetos blancos, decentes y trabajadores (Chambers 2003: 9, 264-268, 280; Jorge Bedregal, comunicación personal, 2020).

La vinculación con lo afro hace uso de formulaciones que evocan un discurso de corte biológico y enciclopédico, y se expresa mediante palabras raciales explícitas como negro, moreno, zambo y mulato:

(5) Tienen una estructura física de negro (Arequipa, ocupación no básica, L14H(57)CNB).

(6) Tienen esa fisonomía... Es que sí, pues, es que son distintos. Ellos tienen otra fisonomía más trigueña, medio mulata. La mayoría sí son de esa fisonomía mulata, caribeña, así (Arequipa, ocupación no básica, C11H(36)BRNB). 
(7) Por genética son más altos, las venezolanas también tienen otro cuerpo, no son delgadas, su estructura ósea no es delgada, es un poco ancha, tanto del venezolano. [...] Ellos tienen descendencia morena, claro (Lima, ocupación básica, $\mathrm{A} 04 \mathrm{H}(31) \mathrm{LOB})^{3}$

La alusión al origen afro también puede codificarse mediante retóricas no explícitamente raciales, pero que han sido asociadas históricamente a la negritud en el Perú: por ejemplo, el detalle en las características del cabello y la animalización (para este último aspecto, ver Velázquez 2005: 79; Gonzales 2018), como vemos en (8) y (9). Este tipo de repertorios, que se suman a los anteriores y no aparecen de manera aislada, solo se presentan en las entrevistas de Arequipa.

(8) ...los chicos son así como medio morenos, zambos que se lacean el cabello la mayoría, no son lacios, todos son como carnero (Arequipa, ocupación básica, C01M(46)CB).

(9) Por su tipo; o sea, pueden ser blancos, pero tienen de todas maneras un tipo, una estructura física de negro. [...] Pero, sí, tú los ves y tienen una cara de monito (ríe) (Arequipa, ocupación no básica, L14H(57)CNB).

Es recurrente el establecimiento de un continuo que va de lo blanco a lo negro para caracterizar la fisonomía de los migrantes; un discurso común es que existen dos tipos muy marcados: los blancos y los negros, junto con sus diferentes variantes, algunas ya mencionadas. El contraste entre este continuo y la fisonomía implícita de los peruanos, codificado con estructuras comparativas como “son más altos” en (7), sugiere que lo que no se está percibiendo en los migrantes es la presencia de lo indígena, que caracterizaría al Nosotros de la sociedad receptora, especialmente en Arequipa:

(10) Creo que son dos tipos, son bien morenos o son bien blancos, no como un peruano que a veces estamos mezclados de todo; pero ellos son medios blancones o morenos... (Arequipa, ocupación básica, L04H(45)CB).

(11) Hablamos de gente también morena, trigueña, otros también de tez blanca pero mulatos, cruce de blancos con negro. La conformación de espigados en algunos casos, la talla también más alta en algunos casos de por medio. O sea, se distinguen claramente de lo que somos... Yo me califico de cholo, yo soy cholo, cholo arequipeño (Arequipa, ocupación no básica, L15H(54)BRNB). 
En el ejemplo (11), la contigüidad entre el señalamiento de una distinción entre los venezolanos y "lo que somos" nosotros, por un lado, y la autocalificación reiterada de "cholo", por otro, ilustra nuestra propuesta. La relación se observa de manera más explícita en el siguiente fragmento, en que el entrevistador preguntó al entrevistado si puede identificar a un venezolano:

(12) Realmente no, porque... Ah, bueno, al menos que compares a una persona de raza netamente peruana con un venezolano. Como ellos no tienen acceso a la parte de la cordillera ni nada, como Chile, Argentina y Perú, entonces no tienen la raza que es nativa de la parte de acá, de la parte central de la cordillera (Arequipa, ocupación no básica, L13H(28)CNB). ${ }^{4}$

Enfatizar la construcción de un Nosotros mestizo-indígena y representar a un Otro venezolano caracterizándolo con rasgos afroamericanos o blancos sugiere que, en la sociedad receptora, se tiende a un borrado de lo afro para caracterizar al Nosotros (Irvine y Gal 2000).

Si bien la polarización entre lo blanco y lo negro y la formulación de dos grandes tipos es la tendencia más marcada en nuestros datos, algunas entrevistas se distancian de este patrón. Por ejemplo, la siguiente entrevistada niega la característica de blancos para estas personas y propone el adjetivo "bronceados":

(13) ...generalmente tienen ojos grandes, labios gruesos, cabello castaño, a veces un poquito más oscuro pero castaño. La piel... no son blancos, ninguno es blanco. Todos son bronceados, de ese tipo... (Lima, ocupación no básica, A16M(49)MNB). ${ }^{5}$

En cualquier caso, parece claro que la retórica racial desempeña un papel importante en los discursos sobre la fisonomía de los migrantes en las dos ciudades estudiadas.

\subsection{La cultura de la belleza: referencias al cuidado personal}

La mayoría de los entrevistados reconocen la existencia de una cultura de la belleza y una vestimenta cuidadosa entre los y las migrantes venezolanas.

(14) ...entonces, en Venezuela o sea hay una cultura de... es una cultura de ser bellos, bellas [...] o sea es una cultura que llevan ellos de verse bien (Arequipa, ocupación básica, L08H(29)BRB). 
(15) ...como que una cultura más allegada al físico, que puede ser malo o bueno (Lima, ocupación básica, A09H(44)MNB).

A primera vista, la existencia de esta cultura se percibiría como una ventaja que tienen los migrantes y de la que carecen los miembros de la sociedad receptora. Se trata de una autorrepresentación negativa en el terreno estético generalizada en nuestros datos, sobre la que volveremos en la sección 6. Ahondemos en este punto. El mismo entrevistado del ejemplo (14) comenta:

(16) ...los peruanos seremos chamba y todo, pero no somos bellos; las mujeres igualmente. Los peruanos, aparte, sí somos descuidados, la mayoría somos descuidados; no somos de estar en el corte [de cabello]. Por ejemplo, yo te entro cada veinte días a la peluquería, ¡Y eso, todavía!; en cambio, el venezolano, por ellos creo que estarían todos los días en una peluquería, verse bien, estar ahí. Nosotros no, definitivamente (Arequipa, ocupación básica, L08H(29)BRB).

Sin embargo, como anuncia el ejemplo (15), existe una ambivalencia en la percepción de esta tendencia ("puede ser malo o bueno"). En (17) y (18) se puede observar esta tensión en una contraposición, codificada por el conector de contraste pero, entre el interés de los migrantes por la estética, expresado con verbos volitivos ("Les importa mucho su apariencia”) y estructuras de modalidad deóntica ("Siempre tienen que andar muy bien vestidos”), con la realidad de una situación económica precaria. Es llamativo que este discurso aparezca entre los entrevistados de ocupación no básica, de economía más holgada:

(17) ...ellos quizás no pueden tener para comer, pero siempre tienen que andar muy bien vestidos (Lima, ocupación no básica, P11M(33)LONB).

(18) ...aparte que se arreglan, se visten bien, se ven pobres, pero están con sus zapatillas Adidas, les importa mucho su apariencia (Arequipa, ocupación no básica, C04M(30)BRNB).

El siguiente fragmento de entrevista con el dueño de una ferretería brinda un ejemplo adicional de esta tensión:

a. Luis. Y usted decía que nosotros somos más criollos. ¿A qué se refiere con "más criollos"?

b. Entrevistado. Hablando, primeramente hablando, y la forma de vestirnos también, a ellos les gusta estar un poco 
más... ¿Cómo se dice? Nosotros para el trabajo, así como me ve, pero ellos quieren estar...

c. Luis. El señor está con su buzo y su polo de deporte, y sus zapatillas.

d. Entrevistado. Por el trabajo...

e. Luis. Bien a las Nike, ¿ah?

f. Entrevistado. Je, por el trabajo. En cambio, ellos no quieren ensuciarse.

(Arequipa, ocupación básica, L10H(43)BRB)

El argumento desarrollado en (19) podría resumirse de la siguiente manera: 1) los peruanos nos vestimos para el trabajo (como se enfatiza en tres ocasiones); 2) el trabajo ensucia; 3) los venezolanos prefieren estar bien vestidos a ensuciarse. De esta argumentación, se puede colegir que las prioridades están invertidas entre peruanos y venezolanos, lo que genera una evaluación moral negativa de los migrantes. Las implicaturas y presuposiciones, señalan Bucholtz y Hall (2005), juegan un rol importante en los procesos indexicales, elementos claves en la construcción identitaria. Cobra sentido, entonces, la posibilidad de interpretar el insistente uso de verbos volitivos (como "gustar" en (19) e "importar" en (18)) y en modalidad deóntica para codificar estas prácticas como una forma de remarcar el carácter consciente y calculado que tienen. Así, en la identidad venezolana que se va construyendo acumulativamente, la estética termina ocupando un lugar saltante frente a la ética, es decir, los mandatos laborales y productivos, representados como los valores principales para los miembros de la sociedad receptora, los "peruanos chamba”, como señaló el entrevistado del ejemplo (16), utilizando el americanismo chamba en su acepción de 'trabajador'.

Encontramos el mismo esquema discursivo en una representación del cuidado puesto por las mujeres migrantes en mejorar su cuerpo. Esta vez el contraste se formula entre los valores estéticos, por un lado, y la formación profesional e intelectual, el "cerebro", sumada a la preparación para la vida familiar, por otro. La entrevistada, una entrenadora física y presentadora de televisión, asocia la cultura de Venezuela con la de Colombia, donde ha vivido antes.

(20) Acá la peruana se prepara: entra a la universidad, lo primero que hace es ir a la universidad, salir profesional, ser una buena ama de casa, ser profesional, ayudar a su familia, crecer, pero allá, la mentalidad en Colombia, Venezuela, no. Ni bien tienes trece, catorce, quince años, lo primero que hacen es: tienen su nariz fea, su primer regalo: operación de nariz. Eres plana, tu primer regalo: tu prótesis. Ellos ven así, tienen esa cultura. No 
se preparan de cerebro la mujer. Más se prepara en belleza que en cerebro (Arequipa, ocupación no básica, C05M(42)BRNB).

Si bien el ejemplo (20) proviene de una entrevistada que puede ser descrita como outlier, al mostrar una postura radicalmente negativa frente a la migración venezolana, lo que no es frecuente en el material, el esquema discursivo que utiliza es el mismo del ejemplo (19), proveniente de un entrevistado que, por el contrario, evidenció mucha apertura ante los migrantes. Ello, sumado a los discursos ejemplificados en (17) y (18), nos lleva a proponer que esta evaluación negativa de la cultura de la belleza constituye un patrón en nuestros datos. Este patrón es más explícito en los entrevistados de ocupaciones no básicas, y tiende a estar más implícito en el otro grupo laboral.

En relación con el ejemplo (20), no se puede dejar de lado la frecuencia con que aparece en nuestras entrevistas el tópico de las migrantes venezolanas como mujeres liberales y exuberantes, tal vez como expresión de una mirada hipersexualizada que ya se viene identificando en algunos acercamientos preliminares a esta migración (Solórzano 2019; Muñoz-Pogossian 2019). Lo ilustra el siguiente ejemplo:

(21) [Hablando de una venezolana] era [de] esas, pues, que andan con las joyas, rubia, bien pintada y todo [...]. Las gorditas de allá son más de usar cosas mucho más pegadas que acá, son más [...], claro, son más liberales. Las de acá creo que son... la gente es más recatada (Lima, ocupación básica, A12H(40)MB).

Liberales parece ser, en el terreno de la presentación personal, el equivalente de voluptuosas en el campo de la fisonomía. Tal como las evaluaciones negativas de la cultura de la belleza, esta representación de las venezolanas ilustra que, al hablar sobre la estética, los miembros de la sociedad receptora pueden verbalizar evaluaciones de otro tipo, más bien vinculadas a la ética de los migrantes. Algo similar podremos ver en la siguiente sección.

\section{Resultados: rasgos asociados al comportamiento}

En los discursos de los entrevistados, también se evidenciaron representaciones sobre las y los migrantes venezolanos asociadas al comportamiento. Primero, discutiremos los discursos según los cuales los venezolanos se caracterizarían por ser bulliciosos. Segundo, analizare- 
mos las referencias a la característica de ser altaneros y soberbios. Por último, revisaremos rasgos valorados más positivamente: ser alegres y extrovertidos.

\section{1. "Fiesta a puerta abierta": la representación de los migrantes como bulliciosos}

Los discursos de los entrevistados establecen una relación entre los migrantes y la característica de ser bulliciosos. Este rasgo se aplica tanto a su forma de hablar como a ciertas prácticas referidas al hecho de que les gusta festejar.

Sobre la producción lingüística de los venezolanos, los participantes indicaron que existe una diferencia en su volumen de voz respecto de los peruanos. La forma verbal más usada para señalar estas diferencias fue hablar con adjetivos de evaluación negativa como fuerte y escandaloso acompañados de enfatizadores como muy o atenuadores como medio.

(22) Medio escandalosos hablan, pues (Lima, ocupación básica, P07H(56)LOB).

(23) Es un fastidio lógicamente, porque uno quiere estar un domingo tranquilo y ahí los escuchas, porque lamentablemente ellos hablan muy fuerte, entonces genera un fastidio, ya no puedes estar un domingo tranquilo en casa (Lima, ocupación no básica, $\mathrm{P} 11 \mathrm{M}(33) \mathrm{LONB})$.

La hablante de (23) califica la situación de "fastidio", sustantivo acompañado por el modalizador categórico "lógicamente”. A la vez, justifica esta calificación con una estructura argumentativa que incluye el uso de conectores causales ("porque") y de consecuencia ("entonces"), lo que le permite validar su razonamiento de modo que no queda lugar para cuestionamientos. También, se puede observar el uso del adverbio evaluativo "lamentablemente". El uso de los adverbios señalados ("lógicamente" y "lamentablemente") puede entenderse como la expresión de asunciones que parten de lo que se entiende como un common ground. Fairclough (2003: 55) señala que las asunciones son de importancia social, porque la idea de comunidad se forma sobre la base de significados compartidos que no se cuestionan. En ese sentido, lo anterior permite a la hablante reforzar su argumento.

Este tipo de valoración negativa de las personas que hablan fuerte no parece ser exclusiva de este grupo en la percepción de los peruanos. Pancorbo, Espinoza y Cueto (2011) analizan representaciones estereo- 
típicas en el Perú a partir de encuestas y entrevistas a residentes del Callao, provincia vecina de Lima, donde los participantes se identifican en menor medida con el grupo de afrodescendientes que con otros y es a este grupo al que menos valoran. Aunque los autores no explicitan que se observa como un rasgo negativo, los participantes identifican a los afroperuanos por hablar fuerte (2011: 333). A pesar de que, en nuestro corpus, la dimensión racial no se observa de forma tan clara en los comentarios sobre el volumen de voz, sí se pueden encontrar alusiones explícitas al físico vinculadas con este rasgo, aunque estas se caracterizan por su vaguedad, mediante determinantes (esa, unos) que orientan la calificación sin terminar de explicitarla:

(24) Si tú eres de Lima sabes, medios alharacosos, gritones, medios bacanes y tienen esa fisonomía (Arequipa, ocupación no básica, C11H(36)BRNB).

(25) [Sobre sus diferencias con los peruanos] Básicamente se los saca por su manera de hablar, porque son muy bulliciosos al hablar y tienen unos rasgos típicos de su conformación física (Arequipa, ocupación no básica, L15H(54)BRNB).

Tanto en (24) como en (25), los dos participantes arequipeños de ocupación no básica hacen referencia a la conformación física de los migrantes venezolanos. Aunque no se explicita el tipo de conformación física, ya se vio en la sección sobre estética que es este mismo grupo el que más relaciona a los venezolanos con la ascendencia afro. Así, podemos sugerir que, en general, existe una evaluación negativa ante la percepción de que los venezolanos hablan con un volumen de voz alto y que, en particular, los arequipeños, sobre todo los de ocupación no básica, parecen establecer una conexión entre la ascendencia afro de los migrantes y la característica de ser bulliciosos. Bucholtz y Hall (2005) explican que, en la construcción identitaria, no solo son relevantes las categorías que circulan dentro del discurso, sino también su yuxtaposición explícita o implícita con otras. Si aceptamos la presencia de una yuxtaposición aquí, estaríamos ante un caso de 'habla racial', en la medida en que se está usando una retórica racial sin hacer alusión a la raza (Zavala y Back 2016: 22). Además, lo anterior parecería ser un caso de 'enregistramiento lingüístico’ (Agha 2003, Rosa y Flores 2017), que naturaliza una relación entre ciertos rasgos lingüísticos y un modelo de persona, el o la migrante venezolana.

El rasgo de bullicioso aparece no solo para caracterizar la forma de hablar de los migrantes, sino también para indicar sus costumbres recreativas, las cuales irían en contra de las desplegadas por el Nosotros. Lo 
anterior sucede de manera más recurrente entre los participantes de Arequipa, como se ve en los siguientes ejemplos. ${ }^{6}$

(26) Nos hemos dado cuenta que tienen otro tipo de modus viven$d i$; ya se ponen en las esquinas, ponen su música, bailan, bailan pero descaradamente, toman ahí afuera, hacen bulla, gritan y todo lo demás. Entonces, como consecuencia de ello y como nosotros no estamos acostumbrados a ese tipo de vida, se ha acordado ya no alquilar a gente venezolana (Arequipa, ocupación básica, L07H(55)BRB).

(27) Sí, que son un poco bulliciosos cuando hacen fiesta, porque acá arriba sí había una pequeña familia y les gustaba hacer fiesta a puerta abierta, entonces, de acá a unas cuadras se sentía toda la bulla, pero como ves, es una zona tranquila; entonces, los vecinos empezaron a quejarse (Arequipa, ocupación no básica, C07M(31)BRNB).

En (26) y (27), se usan los verbos "bailar", "hacer bulla”, "hacer fiesta" con intensificadores, como el adverbio "descaradamente" y la frase "a puerta abierta”, para referir a las acciones que realizan los migrantes. Por otro lado, se emplean formas impersonales ("se ha acordado") y la tercera persona ("los vecinos empezaron a quejarse") para codificar las acciones desarrolladas por algunos arequipeños frente a las molestias causadas por algunos migrantes. Estos usos evitan la implicancia de que los hablantes hayan tomado parte en estas acciones. Se trata de una forma de mitigar el efecto de la declaración y de desvincularse a sí mismos de la evaluación negativa de los migrantes, pero sobre todo de sus consecuencias concretas en la vida social: quejas de los vecinos y restricciones para el alquiler de la vivienda.

Además, se observan conectores de consecuencia para establecer una relación de causa-efecto entre las actividades de los venezolanos y las acciones del Nosotros. Estos conectores tienen el efecto de naturalizar la secuencia de acciones (el carácter bullicioso y las restricciones sociales mencionadas) y proponerla como de sentido común. Plantear los puntos de vista propios como razonables permitiría justificar la visión negativa del Otro (Augustinos y Every 2007 citado en Zavala y Back 2016: 23), en este caso, de los venezolanos. En adición, en (27), el hablante realiza un contraste muy claro entre la población receptora y la migrante: "nosotros no estamos acostumbrados a ese tipo de vida”. Este enunciado se suma a la relación causal para darle más fuerza a la justificación, enfocando las prácticas venezolanas como una transgresión a las normas de Arequipa, caracterizada, por lo que pudimos observar, por patrones de recato bastante marcados en comparación con otras ciudades del Perú. 


\section{2. "Eran Venezuela, pues, el país petrolero": la representación de los migrantes como soberbios y altaneros}

Otra característica negativa sobre la manera de ser de los venezolanos en los discursos de los participantes se asocia con la soberbia y la altanería. Este rasgo ha sido hallado en todos los grupos de entrevistados, aunque los arequipeños lo mencionaron de forma más recurrente. Los adjetivos que se usan son altaneros y soberbios, acompañados de intensificadores y modalizadores.

(28) ...ellos tienen... han tenido por mucho tiempo un rollo de superioridad hacia el resto de países: eran Venezuela, pues, el país petrolero (Lima, ocupación básica, $\mathrm{A} 12 \mathrm{H}(40) \mathrm{MB}$ ).

(29) Sí, altaneritos son, cuando están mareaditos (Arequipa, ocupación básica, L09H(36)BRB).

(30) Yo me acuerdo que, cuando recién comenzaron a llegar acá, a Arequipa, los venezolanos estaban con una actitud media soberbia, como que miraban al cholito de acá, digamos, como algo inferior, algo así (Arequipa, ocupación no básica, $\mathrm{L} 14 \mathrm{H}(57) \mathrm{CNB})^{7}$

El entrevistado de (28) hace uso de la frase "rollo de superioridad" como complemento de "tener". Luego, justifica su idea a través de la frase "eran Venezuela, pues, el país petrolero", la cual se enfatiza mediante el marcador discursivo "pues". Como plantea Zavala (2006: 74), este marcador sirve, en el castellano andino, para realizar inferencias a partir de un enunciado previo que el hablante considera importante para expresar su idea, enfatizándolo. En este caso, el hablante infiere del "rollo de superioridad" de los migrantes el pasado de Venezuela como "país petrolero". En cambio, el participante arequipeño de (29) usa un diminutivo ("altaneritos"). Este uso, que ha sido registrado como característico de la variedad andina de castellano para indicar cortesía (Escobar 2000: 55), podría relacionarse con una función de atenuación. Por su parte, en (30), se utiliza el adjetivo "soberbia" junto al modificador "media" para hacer referencia a la actitud de los migrantes. Esto contrasta con el uso de intensificadores para la característica de bulliciosos del apartado anterior. Como ya se mencionó, al ser la cultura del recato bastante importante en Arequipa, este último rasgo de los migrantes parece ser el que más les choca a los entrevistados, y no tanto el de soberbia. 


\section{3. "Un poquito más sueltos": la representación de los migrantes como amables y alegres}

Los entrevistados también señalaron características asociadas con valoraciones más positivas: una representación compartida por todos los grupos de entrevistados fue que los y las venezolanas se caracterizan por ser alegres y extrovertidos. ${ }^{8}$ Para este rasgo, se usaron estructuras comparativas con el adverbio más, y los enfatizadores muy y bastante fueron los más comunes.

(31) Bueno, son más alegres, algunos son más serviciales, ayudan, pero hay otros que también son bien malandrines (Lima, ocupación básica, $\mathrm{A} 17 \mathrm{H}(31) \mathrm{MB})$.

(32) ...son más carismáticos, más alegres, a veces acá la gente es más seria (Arequipa, ocupación básica, S01M(37)BRB).

(33) ...son personas un poquito más sueltas (Arequipa, ocupación básica_L10H(43)BRB).

En (31) y (32), se pueden observar las comparaciones que se realizan entre los peruanos y los venezolanos. Estos últimos se caracterizan por ser "más alegres” o "más carismáticos” que los primeros. Además, la entrevistada de (32) refiere que la población arequipeña es "más seria”. Sobre esto último, existe una representación generalizada, entre los entrevistados de ocupación básica, de los peruanos como menos extrovertidos que los migrantes. Así, en (33), el entrevistado se refiere a la característica "suelta” con el uso del adverbio "más”, pero, en este caso, con el cuantificador "un poquito”. En (34), el entrevistador le pide más desarrollo al participante sobre esta última característica y, además, describir la manera de ser arequipeña. Al respecto, es importante señalar que el entrevistado es un migrante interno, originario de Lambayeque, una región norteña del Perú, relativamente alejada de Arequipa.

a. Luis. ¿Cómo así “sueltas”?

b. Entrevistado. Al hablar, nosotros somos un poco más reservados. Bueno, según la persona, pero mayormente es así, un poquito más reservados, en cambio ellos [los venezolanos] son un poco más sueltos hablando, te lo dicen de manera directa, más francos, como se dice. $[\ldots]$

c. Luis. Y usted que es del norte, de Monsefú, de Chiclayo, allí es distinto del estilo arequipeño también, ¿̇no? 
d. Entrevistado. Ujum.

$[\ldots]$

e. Luis. ¿Cómo describiría la manera de ser arequipeña?

f. Entrevistado. Acá sí son reservados, son reservados, pero cuando te conocen también te dan ese cariño. (Arequipa, ocupación básica, L10H(43)BRB)

El entrevistado pasa del nosotros al ellos cuando se le pide describir a los arequipeños. Así, en (b), el "nosotros” lo incluye a él y a todos los arequipeños. En cambio, en (f), ante la pregunta del entrevistador, usa el ellos (tácito) para caracterizar a estos últimos. Evidentemente, esto responde al hecho de que el entrevistado no es originario de Arequipa; sin embargo, este fragmento es iluminador, ya que saca a la luz una diferencia en la representación de los arequipeños frente a otros peruanos: aunque ambos serían menos extrovertidos que los venezolanos, se considera que los nacidos en Arequipa son más reservados que el resto de peruanos. Lo anterior muestra cómo el hablante se posiciona de distintas maneras en una misma interacción (Bucholtz y Hall 2005: 593). Se utiliza la repetición de la característica reservado en (f) para codificar esta diferencia, además de la partícula afirmativa "sí" para expresar mayor énfasis. Esta se podría interpretar como un focalizador particularizador, según la propuesta de Ferrari, Giammatteo y Albano (2011: 33), aunque estos autores no incluyen la partícula dentro de su clasificación. Al respecto, González (2016: 801) señala cierto paralelismo tanto sintáctico como semántico entre los focalizadores contrastivos y la partícula sí. De hecho, la segunda acepción del lema sí en el Diccionario de la lengua española recoge su valor contrastivo (Real Academia Española 2014). En ese sentido, este elemento le sirve al hablante para enfatizar el hecho de que los arequipeños son los más reservados dentro del conjunto de peruanos.

En resumen, respecto al comportamiento de los migrantes, los aspectos raciales no dejan de estar presentes. Así, se notan asociaciones (implícitas) entre lo afro y la característica de ser bulliciosos, esto último valorado negativamente. Otra característica negativa es la percepción de soberbia y altanería. Sin embargo, también hubo valoraciones positivas relacionadas con el desenvolvimiento más alegre de los migrantes en comparación con los peruanos.

\section{Discusión}

Quijano, uno de los principales teóricos del enfoque decolonial, definió la colonialidad como un mecanismo originado en América y globaliza- 
do a partir de este continente, basado en clasificaciones raciales-étnicas, que permea toda esfera y dimensión de la existencia diaria de los países que estuvieron sujetos a este tipo de dominación (Quijano 1992, 2014). Es comprensible que en un país poscolonial como el Perú un fenómeno migratorio con la magnitud de la diáspora venezolana pase al discurso a través de categorías y retóricas raciales. Desde una perspectiva construccionista, Zavala y Back (2016: 16) invitan a preguntarnos cómo diferentes aspectos específicos de las personas y los grupos empiezan a racializarse. Lo hemos visto en este trabajo al enfocarnos en las representaciones estéticas y conductuales que la sociedad receptora construye sobre los venezolanos. En mayor o menor grado, la tipificación física de los migrantes y su calificación como bulliciosos pueden entroncarse con retóricas asociadas a la negritud en la historia peruana. Sin embargo, habría que reconocer que no todos los discursos identificados en nuestra data pueden enmarcarse con facilidad en esta perspectiva. Así, aunque en otro trabajo se ha registrado la asociación entre los grupos afrodescendientes y la característica de ser alegres (Pancorbo, Espinoza y Cueto 2011: 334), no se identificó una relación explícita ni implícita al respecto en este estudio.

Al observar las similitudes y diferencias entre Lima y Arequipa, es saltante la mayor propensión de los entrevistados arequipeños tanto a asociar las características estéticas de los migrantes con una ascendencia afro como a autorrepresentarse negativamente, desde el punto de vista estético, en comparación con ellos. Este hecho dialoga con los hallazgos de Kogan y Galarza (2014), quienes identificaron una correlación entre autoidentificación étnica y evaluación estética entre estudiantes universitarios peruanos: en los autodefinidos como blancos, esta correlación era positiva; en los autodefinidos como quechuas y mestizos, la tendencia era la contraria. En las cuatro ciudades estudiadas por ellos (Lima, Arequipa, Huancayo y Trujillo), alrededor de $70 \%$ de los estudiantes entrevistados se percibieron como mestizos, pero solo $13 \%$ de los arequipeños y huancaínos se percibieron como blancos (frente a $19 \%$ en Lima y $17 \%$ en Trujillo).

Si bien esto sugiere que, en el terreno estético, los miembros de la sociedad receptora se posicionan por debajo de los migrantes debido a la valoración negativa del factor indígena, al mismo tiempo podemos ver que se percibe a los migrantes como parte de un continuo entre lo blanco y lo negro que niega la presencia de lo indígena en ellos. De la misma forma, la autorrepresentación del Nosotros está borrando, en el sentido de Irvine y Gal (2000), lo afroperuano, lo cual es especialmente notorio en Arequipa. Se relaciona con este hallazgo la autopercepción histórica de Arequipa como una Ciudad Blanca, marcada por criterios de decencia y recato que también permiten explicar el mayor énfasis en la repre- 
sentación de los migrantes como bulliciosos. Aunque las diferencias halladas entre los discursos imperantes en las dos ciudades son solo de grado, ellas muestran la importancia de una investigación situada, que tome en cuenta la cultura y la historia local.

Una perspectiva economicista en torno a los discursos sobre la migración predeciría que los trabajadores que desempeñan ocupaciones básicas serían quienes desplieguen discursos más negativos en torno a los migrantes y viceversa. En contraste, nosotros hemos encontrado que, en relación con la descripción física y la evaluación moral de la cultura de la belleza, estos discursos son más claros entre los trabajadores de ocupaciones no básicas. Además, la caracterización positiva como amables y alegres fue identificada en todos los grupos de entrevistados. Así, los fundamentos raciales parecen explicar mejor algunos de los discursos identificados que los determinantes laborales y económicos.

Como una limitación del estudio, debemos resaltar que nuestra recolección de datos corresponde al periodo previo a la pandemia por COVID-19, en un momento temprano en la historia de esta diáspora en el Perú. Futuras investigaciones podrán abordar los posibles cambios respecto al panorama descrito en momentos de mayor estabilización de la población migrante y tomando en cuenta los efectos negativos de la pandemia.

De cualquier forma, esperamos haber comprobado la utilidad de la aplicación de determinadas categorías de análisis lingüístico en el marco de los nuevos estudios de racialización y de la lingüística sociocultural para explicar un proceso migratorio sin precedentes en el país receptor. Así, algunas estrategias discursivas encontradas para la representación de los migrantes -modalización categórica para el encomio estético y la codificación del carácter bullicioso, tipos de verbos específicos para hablar sobre la cultura de la belleza y estructuras de causa-consecuencia y formas despersonalizadas para legitimar discursivamente la exclusión y la restricción de derechos- cobran un sentido más preciso si se las observa en el marco de la reproducción de jerarquías basadas en lo étnico-racial y en el de la construcción identitaria a través del discurso.

\section{Conclusión}

Nuestros hallazgos indican que los aspectos raciales son importantes para la caracterización de los migrantes venezolanos no solo en cuanto a sus aspectos físicos sino también conductuales. Aunque esta retórica racial no es explícita en estos últimos, como sí ocurre con el plano estético, se han observado claras relaciones con lo afro para la característica de bulliciosos. Esto sugiere que la racialización a través del lenguaje y la 
construcción de la diferencia en la interacción constituyen enfoques útiles para analizar los discursos sobre los fenómenos migratorios Sur-Sur en países poscoloniales, sin que ello signifique que no sean pertinentes también para otros contextos de migración. Después de todo, como recuerdan Rosa y Flores (2017: 622, traducción nuestra), el mundo actual "está profundamente modelado por la globalización del colonialismo europeo", por lo cual "las particularidades y los patrones raciolingüísticos deben ser entendidos en todo el mundo moderno”.

Luis Andrade Ciudad

Pontificia Universidad Católica del Perú

Av. Universitaria 1801, San Miguel,

Lima 32, Perú

lfandrad@pucp.edu.pe

ORCID: 0000-0001-7270-9033

Sergio Lazarte Chicata

Universidad Nacional de San Agustín

de Arequipa

Av. Independencia, s. n, Cercado,

Arequipa, Perú

ORCID: 0000-0002-9711-5191

Pamela Jimenez Lizama

Pontificia Universidad Católica del Perú

Av. Universitaria 1801, San Miguel, Lima 32, Perú

ORCID: 0000-0002-9817-5946

Recepción: 17/11/2020; Aceptación: 15/07/2021
Anahís Samamé Rispa

Pontificia Universidad Católica del Perú

Av. Universitaria 1801, San Miguel,

Lima 32, Perú

ORCID: 0000-0002-7079-3873

Claudia Crespo del Río

Pontificia Universidad Católica del Perú

Av. Universitaria 1801, San Miguel,

Lima 32, Perú

ORCID: 0000-0002-3851-2023

\section{Notas}

1 Los Olivos es un distrito de la zona norte de Lima, mientras que Magdalena pertenece a la zona centro de la ciudad; ambos albergan gran cantidad de migrantes (OIM 2018). Para los distritos de Arequipa, la selección se realizó a partir del conocimiento de la ciudad de uno de los investigadores, quien reside en ella. Agradecemos al antropólogo Jorge Bedregal, de la Universidad Nacional San Agustín de Arequipa, por iluminadoras conversaciones sobre etnicidad y cultura arequipeña; al lingüista Héctor Velásquez, de la Pontificia Universidad Católica del Perú, por ayudarnos a aclarar conceptos sobre foco y estructura informativa; a la antropóloga Liuba Kogan, de la Universidad del Pacífico, por útiles sugerencias bibliográficas; y al empresario Germán Ordóñez Rodríguez, el lingüista Jorge Iván Pérez, el ingeniero Gino Corrales, el profesor Henry Rivas, el administrador Adolfo Villanueva y la lingüista Mélida Conislla por habernos facilitado generosamente la recolección de datos.

2 Sin embargo, el distrito arequipeño de Acarí, en la provincia de Caravelí, tradicionalmente se reconoce como una localidad con presencia afroperuana, lo que se refleja en el censo $(6,28 \%, 248$ personas). Para datos históricos sobre la presencia de afroperuanos en Arequipa, ver Chambers (2003: 92-96, 102-104). 
3 El entrevistado dice "descendencia morena" en vez de "ascendencia morena". "Moreno" es uno de los términos históricamente asociados con la ascendencia afro en el Perú.

4 Esta percepción se basa en la idea geográfica errónea de que la cordillera de los Andes no tiene relación alguna con Venezuela, cuando en realidad se origina allí.

5 En el mismo sentido, pero con contenido inverso, una entrevistada arequipeña niega categóricamente la característica de negros para estos migrantes: "Ellos tienen la piel blanca y no he visto venezolanos negros, no he visto; he visto blancos" (Arequipa, ocupación básica, L02M(52)CB).

6 Llama la atención, en los ejemplos (26) y (27), la asociación entre la idea de fiesta y la ocupación del espacio ("se ponen en las esquinas", "toman ahí afuera", "hacer fiesta a puerta abierta”). Sería interesante profundizar en esta relación. Es sugerente la idea de que existe un repertorio interpretativo más amplio, que integra el carácter bullicioso, la expansión en el espacio público y el ser "un poco más sueltos", como veremos después. Proponemos considerar esta línea de investigación en trabajos futuros.

7 Nótese el diminutivo atenuador en la palabra racial "cholito".

8 La asociación entre ser alegres y afrodescendientes ha sido registrada por Pancorbo, Espinoza y Cueto (2011:333). En las entrevistas realizadas en nuestro estudio, este nexo no se ha evidenciado. Sin embargo, al haber referencias a la ascendencia afro de los migrantes en relación con otros aspectos, sería importante profundizar en este vínculo.

\section{Referencias bibliográficas}

Agencia EFE. 2018. "Daniel Alarcón, creador de 'Radio Ambulante': la historia es ahora Venezuela". https://www.efe.com/efe/america/ cultura/daniel-alarcon-creador-de-radio-ambulante-la-historia-ahora -es-venezuela/20000009-3556166 [Consulta: 28/08/2020].

Agha, Asif. 2003. "The social life of cultural value". Language $E$ Communication, 23. 231-273.

Albelda, Marta. 2018. "Variación sociolingüística de los mecanismos mitigadores: diferencias de uso en edad y sexo". Cultura, Lenguaje $y$ Representación, 19. 7-29. http://dx.doi.org/10.6035/clr.2018.19.1

Anderson, Kate. 2008. "Justifying race talk: Indexicality and the social construction of race and linguistic value". Journal of Linguistic Anthropology, 18: 1. 108-129.

Arellano, Rolando y David Burgos. 2010. Ciudad de los Reyes, de los Chávez, de los Quispe... Lima: Arellano Marketing / Planeta.

Blommaert, Jan. 2010. The sociolinguistics of globalization. Nueva York: Cambridge University Press.

Blouin, Cécile (dir.). 2019. Estudio sobre el perfil socio económico de la población venezolana y sus comunidades de acogida: una mirada hacia la inclusión. Lima: Fundación Panamericana para el Desarrollo / IDEHPUCP.

Briz, Antonio. 2006. "Para un análisis semántico, pragmático y sociopragmático de la cortesía atenuadora en España y América”. Lingüistica Española Actual, 29:1. 5-40. 
Bucholtz, Mary y Kira Hall. 2005. "Identity and interaction: a sociocultural linguistic approach”. Discourse Studies, 7:4-5. 585-614.

Chambers, Sarah. 2003. De súbditos a ciudadanos: honor, género y politica en Arequipa, 1780-1854. Lima: Red para el Desarrollo de las Ciencias Sociales en el Perú.

Escobar, Anna María. 2000. Contacto social y lingüístico. El español en contacto con el quechua en el Perú. Lima: Fondo Editorial de la PUCP.

Franco, Carlos. 1986. Exploraciones en "otra modernidad": de la migración a la plebe urbana. Lima: CEDEP.

Fairclough, Norman. 2003. Analysing discourse. Textual analysis for social research. Londres: Routledge.

Ferrari, Laura, Mabel Giammatteo e Hilda Albano. 2011. "Operadores de foco: el caso de incluso, hasta, solo y aun". Cuadernos de la ALFAL 3. 30-41. https://mundoalfal.org/sites/default/files/ revista/03_cuaderno_005.pdf [Consulta: 28/09/2020].

González, Raquel. 2016. "Polaridad: afirmación y negación”. En Enciclopedia de Lingüística Hispánica, Javier Gutiérrez-Rexach (coord.), 797-808. Volumen 1. Londres: Routledge.

Gonzales, Sharún. 2018. “CCómo estudiar el racismo en la prensa deportiva?”. Conexión, 7:10. 85-97.

Instituto Nacional de Estadística e Informática. 2017. Censos Nacionales 2017: XII de Población, VII de Vivienda. Sistema de consulta de base de datos. https://censos2017.inei.gob.pe/redatam/ [Consulta: 30-06-2020].

Instituto Nacional de Estadística e Informática. 2016. Clasificador nacional de ocupaciones 2015. Lima: INEI.

Irvine, Judith T. y Susan Gal. 2000. "Language ideology and linguistic differentiation". En Regimes of language: Ideologies, polities, and identities, Paul V. Kroskrity (ed.), 35-84. Santa Fe: School of American Research Press.

Kesselheim, Wolfgang. 2007. "Dos maneras de construir el rechazo a la inmigración en el discurso migratorio de la Argentina”. Revista Internacional de Lingüistica Iberoamericana, 2:10. 127-146.

Koechlin, José, Ximena Solórzano, Giovanna Larco y Enrique Fernández-Maldonado. 2019. Impacto de la inmigración venezolana en el mercado laboral de tres ciudades: Lima, Arequipa y Piura. Lima: OIM / OIT / Ministerio de Trabajo / Universidad Antonio Ruiz de Montoya.

Kogan, Liuba y Francisco Galarza. 2014. ¿Discriminas o te discriminan? Un análisis de las percepciones de universitarios de cuatro ciudades del Perú. Lima: Universidad del Pacífico. 
León, Eduardo y María del Carmen Falcón. 2016. “Migración Sur-Sur: factores relacionales e inserción segmentada de la población boliviana y peruana en la ciudad de Córdoba, Argentina”. Estudios Demográficos y Urbanos, 31:3. 279-773.

Loayza, Jerjes. 2020. "Inmigración venezolana y estigmatización laboral en el Perú”. Investigaciones Sociales, 23:43. 179-192. http://dx.doi.org/10.15381/is.v23i43.18492

Matos Mar, José. 1984. Desborde popular y crisis del Estado: el nuevo rostro del Perú en la década de 1980. Lima: Instituto de Estudios Peruanos.

Muñoz-Pogossian, Betilde. 2019. "Migrating as a Venezuelan woman”. Caracas Chronicles. 8 de marzo. https:/www.caracaschronicles.com/ 2019/03/08/migrating-as-a-venezuelan-woman/ [Consulta: 20-092020].

OECD. 2011. "Inmigrant integration in the South". En Tackling the Policy Challenges of Migration: Regulation, Integration, Development. OECD Publishing. https://dx.doi.org/10.1787/ 9789264126398-7-en

Organización Internacional para las Migraciones. 2018. Monitoreo de flujo de la población venezolana en el Perú. DTM Ronda 3. Lima: OIM.

Organización Internacional para las Migraciones / UNICEF. 2020. Monitoreo de flujo de la población venezolana en el Perú. DTM Reporte 7. Lima: OIM.

Palmer, F. R. 2001. Mood and modality. Cambridge: Cambridge University Press.

Pancorbo, Gina, Agustín Espinoza y Rosa María Cueto. 2011. "Representaciones estereotípicas y expresión del prejuicio en el Perú: la mirada desde la pobreza”. Revista de Psicología, 29: 2. 311342. https://doi.org/10.18800/psico.201102.006

Quijano, Aníbal. 1992. "Colonialidad y modernidad/racionalidad". Perú Indígena, 13: 29. 11-20.

Quijano, Aníbal. 2014. "Colonialidad del poder y clasificación social”. En Cuestiones y horizontes. De la dependencia histórico-estructural a la colonialidad/descolonialidad del poder, Aníbal Quijano, 285-327 [Selección de Danilo Assis Clímaco]. Buenos Aires: CLACSO.

Real Academia Española. 2014. Diccionario de la lengua española, 23. ${ }^{a}$ ed., [versión 23.3 en línea]. https://dle.rae.es [Consulta: 18/08/2020].

Rosa, Jonathan y Nelson Flores. 2017. "Unsettling race and language: Toward a raciolinguistic perspective". Language in Society, 46. 621-647. URL: doi:10.1017/S0047404517000562

Solórzano, Ximena. 2019. “Las más vulnerables entre los desiguales”. Revista Ideele, 287. https://revistaideele.com/ideele/content/ 
las-m\%C3\% A1s-vulnerables-entre-los-desiguales [Consulta: 1508-2020].

Velázquez, Marcel. 2005. Las máscaras de la representación: el sujeto esclavista y las rutas del racismo en el Perú (1775-1895). Lima: UNMSM / BCR.

Wiemer, Björn. 2018. "Evidentials and epistemic modality". En The Oxford handbook of evidentiality, Alexandra Y. Aikhenvald (ed.). Oxford: Oxford University Press.

Zavala, Virginia. 2006. "Transferencia de funciones evidenciales del quechua: el rol de pues como marcador discursivo en el español andino". Lexis, 30:1. 55-82. URL: http://revistas.pucp.edu.pe/index.php/ lexis/article/view/1195 [Consulta: 30-09-2020].

Zavala, Virginia y Michele Back. 2016. "Introducción: la producción discursiva de identidades racializadas". En Racismo y lenguaje, Virginia Zavala y Michele Back (eds.), 11-38. Lima: Fondo Editorial de la Pontificia Universidad Católica del Perú. 